\title{
On the nature of optical oscillations on the flare stars
}

\author{
E. G. Kouprianova ${ }^{1}$, Y. T. Tsap ${ }^{2}$, Y. G. Kopylova ${ }^{1}$ and \\ A. V. Stepanov ${ }^{1}$ \\ ${ }^{1}$ Central Astronomical Observatory, Pulkovo, Russia, email: lioka@gao.spb.ru \\ ${ }^{2}$ Crimean Astrophysical Observatory, email: yur@crao.crimea.ua
}

\begin{abstract}
On the basis of an analogy between solar and stellar flares the optical oscillations with periods of tens of seconds observed on the EV Lac are analyzed. In terms of the diagnostic tool proposed by Zaitsev \& Stepanov (1982) the main parameters of the flare loop was obtained. We concluded that source of the optical radiation is located at the loop footpoints and its pulsations are determined by the modulation of the flux of energetic particles.
\end{abstract}

Coronal magnetic arches (loops) are the principal structural elements of upper atmospheres of the Sun and red dwarfs (Gershberg 2002). According to the widely accepted scenario of solar flares, a coronal arch, which can be regarded as the magnetic trap, accumulates accelerated particles. Trapped particles are scattered into the loss-cone and heat the dense chromospheric plasma, the hottest part of which with $T \sim 10^{7}-10^{8} \mathrm{~K}$ is evaporated and fills a loop, emitting in the soft X-rays. Thus, coronal loops determine the peculiarities of thermal and nonthermal emission of a flare. In contrast to the solar flares, direct observations of the stellar flare loops are impossible due to large distances. However this problem can be resolved if we take into account the solar-stellar analogy.

In this report we connect the flare oscillations on EV Lac with periods $P=10-30 \mathrm{~s}$ observed in the $U$ and $B$ bands (Zhilyaev et al. (2000)) with the fast magnetoacoustic (FMA) oscillations of a loop. On the basis of the model proposed by Zaitsev \& Stepanov (1982), the parameters of the flare plasma are estimated. A problem of localization of the source of optical emission is discussed.

Taking into account that, for solar and stellar coronae, the plasma parameter $\beta=$ $8 \pi p / B^{2} \ll 1$, the amplitude of disturbance of the magnetic field $\delta B$ caused by increasing of the gas pressure $p$ inside a flare loop can be found from the following equation

$$
p+\frac{B \delta B}{4 \pi}=0
$$

Period of the fundamental harmonic of the leaky FMA modes is

$$
P=2 \pi a /\left(\eta_{0} v_{r}\right), \quad v_{r}=\sqrt{v_{A}^{2}+c_{s}^{2}}
$$

where $a$ is the radius of a loop cross section, $v_{A}=B / \sqrt{4 \pi n M}$ and $c_{s}=\sqrt{5 / 3 T / M}$ are the Alfven and sound velocities, respectively, $\eta_{0} \approx 2.4$ is the first zero of the Bessel function $J_{0}(\eta)$.

Analysis shows that the damping of the FMA oscillations of the coronal loops is determined by the ion viscosity $(v)$ and thermal electron conductivity $(c)$. Therefore the total decrement can be written (Stepanov et al. (2004)) as

$$
\gamma_{\Sigma}=\gamma_{v}+\gamma_{c}=\frac{1}{3} \sqrt{\frac{M}{m}} \frac{\omega^{2}}{\nu_{e i}} \beta \sin ^{2} \theta\left(\frac{1}{4 \sqrt{2}}+\sqrt{\frac{M}{m}} \beta \cos ^{2} \theta\right) .
$$


Here $M$ and $m$ are the ion and electron masses, $\theta=\arctan \left(k_{\perp} / k_{\|}\right)$is the angle between the direction of the magnetic field $\mathbf{B}$ and the wave vector $\mathbf{k}$, and the electron-ion frequency of collisions $\nu_{e i} \approx 60 n / T^{3 / 2} \mathrm{~s}^{-1}$.

The Q-factor of the oscillations can be represented as $Q=\omega / \gamma_{\Sigma}$. Then, combining equations (1)-(3), we can derive the formulae for determining the flare plasma parameters

$$
\begin{gathered}
T \approx 1.2 \times 10^{-8} \frac{\tilde{r}^{2} \beta}{P^{2} \chi}, \quad n \approx 3.49 \times 10^{-13} \frac{\tilde{r}^{3} \kappa \beta^{5 / 2} Q \sin ^{2} \theta}{P^{4} \chi^{3 / 2}}, \\
B \approx 3.81 \times 10^{-18} \frac{Q^{1 / 2} \tilde{r}^{5 / 2} \kappa^{1 / 2} \beta^{5 / 4} \sin \theta}{P^{3} \chi^{5 / 4}},
\end{gathered}
$$

where $\tilde{r}=2 \pi a / \eta_{0}, \kappa=243 \beta \cos ^{2} \theta+1, \chi=10 / 3 \beta+2$.

In order to find out the dependence between the modulation depth of optical emission $\Delta=\left(F_{\max }-F_{\min }\right) / F=2 \delta F / F$, where $F_{\max }=F+\delta F$ and $F_{\min }=F-\delta F$ are the maximum and minimum spectral flux densities of the modulated emission, respectively, and plasma parameter $\beta$, we assume that the pulsations of optical emission are generated by the modulated flux of precipitated electrons at the footpoints of a flare loop. In case of the moderate or strong diffusion regimes (Stepanov \& Tsap 2002) the modulation depth of the optical emission from the loop footpoints caused by the FMA oscillations is

$$
\Delta \approx \beta .
$$

As follows from the observations of flares on EV Lac: $P \approx 13 \mathrm{~s}, Q \approx 50, \Delta \approx 0.2$. Then, taking $L / a=10\left(\theta \approx 76^{\circ}\right), \tilde{r}=2.62 a \approx 2.62 \times 10^{9} \mathrm{~cm}$, from (4)-(6) we find the temperature of plasma $T \approx 3.7 \times 10^{7} \mathrm{~K}$, plasma density $n \approx 1.6 \times 10^{11} \mathrm{~cm}^{-3}$, and magnetic field strength $B \approx 320 \mathrm{G}$. Apart from that, the loop length $L \approx 10^{10} \mathrm{~cm}$, i.e. $L \approx 0.4 R_{\star}$.

Mullan et al. (1992) proposed that the flare optical radiation is determined by the free-free emission of the thermal plasma of the coronal portion of a loop. This hypothesis can be considered as an alternative approach. However, it is easy to show that, due to modulation of free-free emission of the coronal plasma by the FMA modes, the modulation depth $\Delta \approx(1.25 \sin \theta-1) \beta$. Therefore, if we take the values of the parameters as in the previous case, we find from (4) and (5): $T \approx 9 \times 10^{7} \mathrm{~K}, n \approx 10^{13} \mathrm{~cm}^{-3}, B \approx 1900 \mathrm{G}$. The obtained results contradict observations as well as some physical considerations.

\section{Acknowledgements}

This study was supported by the INTAS (grant no. 00-543), RFBR (projects no. 0302-17218 and 03-02-17357), the "Astronomy" State Science and Technology Program, the Program of the Presidium of RAS "Nonstationary Phenomena in Astronomy", the Ministry of Industry and Science under grant 40.022.1.1.1104, Administration of SaintPetersburg (project no. PD04-1.9-35.), and the OFN-16 program.

\section{References}

Gershberg, R. E. 2002 Activity of the Solar Type Stars of the Main Sequence, Odessa, Astroprint, 2002 (in Russian).

Mullan, D.J., Herr, R.B. \& Bhattacharyya, S. 1992 Astrophys. J. 391, 265-275.

Stepanov, A.V., Kopylova Yu. G., Tsap Yu. T. et al. 2004 Astron. Lett. 30, 480-488.

Stepanov, A.V. \& Tsap, Y.T. 2002 Solar Phys. 211, 135-154.

Zaitsev, V.V. \& Stepanov, A. V. 1982 Sov. Astron. Lett. 8, 132-134.

Zhilyaev, B. E., Romanyuk, Ya. O., Verlyuk, I. A. et al. 2000 Astron. Astrophys. 364, 641-645. 